\title{
In vitro permeation studies of phenolics from horse chestnut seed gels prepared with different polyacrylic acid polymer derivatives
}

\author{
EGLË ZELBIENE $\dot{H}^{1}$ \\ GAILUTE DRAKSIENE \\ ARUNAS SAVICKAS ${ }^{1}$ \\ DALIA KOPUSTINSKIENE ${ }^{1}$ \\ RUTA MASTEIKOVA ${ }^{2}$ \\ JURGA BERNATONIENE ${ }^{1 *}$ \\ ${ }^{1}$ Lithuanian University of Health \\ Sciences, Medical Academy \\ Faculty of Pharmacy \\ 50009 Kaunas, Lithuania \\ ${ }^{2}$ University of Veterinary and \\ Pharmaceutical Sciences \\ Faculty of Pharmacy \\ 61242 Brno, Czech Republic
}

\begin{abstract}
The aim of this study was to investigate the effects of poly= acrylic acid polymers (Ultrez 10, Ultrez 20, Carbopol 980, and Carbopol 940) on the viscosity and the in vitro permeation of phenolic compounds from the gel prepared from natural horse chestnut seed extract. Experiments were performed in the presence and in the absence of peppermint oil (Mentha piperita). Our results showed that peppermint oil decreased the viscosity of the gels and permeation of phenolic compounds from all gel samples. Results show that the highest content of phenolic compounds $\left(1.758 \mu \mathrm{g} \mathrm{cm}^{-2}\right)$ permeated in vitro from gel based on Carbopol Ultrez 20 without peppermint oil added $(p<0.05$ vs. other tested polymers).
\end{abstract}

Keywords: horse chestnut, polyacrylic acid polymers, permeation

Accepted December 22, 2014

Horse chestnut seeds are known to contain 3-6\% of triterpenoid saponins, flavonoids (mostly quercetin and kaempferol glycosides), tannins and coumarins (in seed coats), vitamins C and B, essential oils, and other substances (sugars, carbohydrates, fatty oils, purines, proteins, and mineral substances), which have a broad spectrum of pharmacological activities $(1,2)$. Flavonoids are characterized by their multi-functional activity and applicability; for example, the well-known anti-radical activity on the skin (3), anti-inflammatory and antimicrobial activity (2). Especially rutin and its derivatives exhibit a protective effect on blood vessel walls (3). A. hippocastanum extracts are used in cosmetic formulations due to the aforementioned composition (4). However, there have been no studies so far on the influence of the base used in the production of semisolid dosage form on the release of active compounds of the horse chestnut seed extract. For this reason, the aim of this work was to investigate the in vitro permeation of phenolics from horse chestnut seed gels prepared using different polyacrylic acid polymer derivatives.

\footnotetext{
* Correspondence; e-mail: jurgabernatoniene@yahoo.com
} 
E. Zelbiene et al:: In vitro permeation studies of phenolics from horse chestnut seed gels prepared with different polyacrylic acid polymer derivatives, Acta Pharm. 65 (2015) 181-189.

\section{EXPERIMENTAL}

\section{Materials}

Polyacrylic acid polymers Ultrez 10, Ultrez 20, Carbopol 980 and Carbopol 940 were all from Lubrizol, USA. Triethanolamine, trifluoroacetic acid, acetonitrile polypropylene glycol, isopropyl alcohol, sodium carbonate and standard gallic acid (97\% purity) were from Sigma-Aldrich Chemie $\mathrm{GmbH}$, Germany.

HPLC grade purity quercetin, kaempferol, astragalin, isoquercetin, leucocyanidin, rutin, quercitrin, epicatechin, proanthocyanidin A2, Folin's reagent and peppermint oil (Mentha piperita) were supplied by Carl Roth (Germany). All chemicals and solvents were of analytical grade.

\section{Preparation of the horse chestnut seed extract}

Experimental studies were carried out on horse chestnut (Aesculus hippocastanum L., Sapindaceae) seeds from the Utena district (Lithuania) collected in autumn 2012. Washed and cleaned seeds were stored for three months in a dark and dry place at the temperature of $25 \pm 2{ }^{\circ} \mathrm{C}$. The cellulosic and wooden hull and red-brown endosperm were hand-peeled from the seeds. The air-dried seeds were ground to powder, then extracted with $70 \%$ ethanol in water using an Ultra-Turrax device (Polytron, Switzerland). The extract was filtered, concentrated under reduced pressure, and then dried. Dry extract was prepared using a spray-dryer Büchi 190 (Polytron) at heating temperature, 161-168 ${ }^{\circ} \mathrm{C}$, output temperature $105-110^{\circ} \mathrm{C}$ and dry extract spraying rate $7.0-8.2 \mathrm{~mL} \mathrm{~min}^{-1}$.

\section{Gel preparation}

The composition of gels used in the study is presented in Table I. During the preparation process, $1 \%$ of polymer was dispersed in water, subsequently adding propylene glycol, isopropyl alcohol, and peppermint oil. Horse chestnut extract was dissolved in water. A filtered aqueous solution was added to the polymer suspension under continuous stirring. The resulting suspension was neutralized with $10 \%$ triethanolamine until pH $7.0 \pm$ 0.2 . Gel homogeneity was achieved by using an automated mixing system »Unguator $2100 \ll$ (GAKO International GmbH, Germany).

\section{Viscosity evaluation}

The test sample was poured into a clean and dry $150-\mathrm{mL}$ beaker and its viscosity was measured with a CAP-2000 Brookfield viscometer (Brookfield Engineering Laboratories, USA) during the standard operating procedure at the temperature of $25 \pm 2{ }^{\circ} \mathrm{C}$ using a spindle 7 and $10 \mathrm{rpm}$, measurement time $30 \mathrm{~s}$.

\section{In vitro permeation through the dialysis membrane}

In vitro permeation testing was conducted by applying a cellulose membrane that was soaked in water at $37 \pm 2{ }^{\circ} \mathrm{C}$ for 24 hours prior to the procedure. A diffusion cell was used where the surface area of the dialysis membrane in contact with the acceptor medium was 
E. Zelbiene et al:: In vitro permeation studies of phenolics from horse chestnut seed gels prepared with different polyacrylic acid polymer derivatives, Acta Pharm. 65 (2015) 181-189.

Table I. Chemical composition of a dry seed extract from the pure species of Aesculus hippocastanum

\begin{tabular}{lr}
\hline Constituent & Content \\
\hline Phenolic compounds $\left(\mathrm{mg} \mathrm{g}^{-1}\right)$ & $61.2 \pm 0.9$ \\
Quercetin $\left(\mu \mathrm{g} \mathrm{mL}^{-1}\right)$ & $85.7 \pm 0.3$ \\
Kaempferol $\left(\mu \mathrm{g} \mathrm{mL}^{-1}\right)$ & $62.0 \pm 0.9$ \\
Astragalin $\left(\mu \mathrm{gL}^{-1}\right)$ & $8.0 \pm 0.1$ \\
Isoquercitrin $\left(\mu \mathrm{g} \mathrm{mL}^{-1}\right)$ & $15.0 \pm 0.3$ \\
Leucocyanidin $\left(\mu \mathrm{g} \mathrm{mL}^{-1}\right)$ & $6.2 \pm 0.2$ \\
Rutin $\left(\mu \mathrm{g} \mathrm{mL} \mathrm{m}^{-1}\right)$ & $34.9 \pm 7.3$ \\
Quercitrin $\left(\mu \mathrm{g} \mathrm{mL}^{-1}\right)$ & $13.0 \pm 0.4$ \\
Epicatechin $\left(\mu \mathrm{g} \mathrm{mL}^{-1}\right)$ & $52.9 \pm 4.1$ \\
Proanthocyanidin $\mathrm{A}_{2}\left(\mu \mathrm{g} \mathrm{mL} L^{-1}\right)$ & $3.4 \pm 0.2$ \\
\hline
\end{tabular}

Mean \pm SEM, $n=3$.

$1.76625 \mathrm{~cm}^{2}$. The acceptor medium was $30 \mathrm{~mL}$ of phosphate buffer (pH 7.30). During the test, $0.5 \mathrm{~g}$ of the studied gel was placed on the membrane. The prepared sample was fixed to ensure contact between the membrane and the acceptor medium. The diffusion cell was placed into a water bath. A heated magnetic stirrer was used to maintain stable temperature $\left(37 \pm 2{ }^{\circ} \mathrm{C}\right)$ and stirring $(600 \mathrm{rpm})$. The testing was carried out for 6 hours, and $0.7-\mathrm{mL}$ samples of the acceptor medium were taken at specified time intervals, being replaced with an qual volume of the buffer solution.

\section{HPLC analysis of the dry seed extract}

HPLC analysis was carried out using the Waters 2690 Alliance HPLC system (Waters Corporation, USA), equipped with a Waters 2487 UV/Vis detector, an on-line degasser and an auto sampler, and a Waters XTerra RP18 150×3.9 mm column. UV detection was achieved at $360 \mathrm{~nm}$. Chromatographic elution was accomplished using a gradient solvent system consisting of water containing $0.1 \%$ trifluoroacetic acid (TFA) (A) and acetonitrile containing $0.1 \%$ TFA (B). Gradient conditions were the following: 0-45 min: $95 \%$ A, $5 \%$ B; 45-50 min: $55 \%$ A, $45 \%$ B, from 55 min: $95 \%$ A, $5 \%$ B at the flow rate of $400 \mu \mathrm{L} \mathrm{min}{ }^{-1}$. The injection volume was $10 \mu \mathrm{L}$. Buffer solutions were filtered through a $0.2-\mu \mathrm{m}$ disposable membrane filter (Roth, Germany), and were degassed prior to use. Data were collected and analyzed using the Waters Millennium $2000^{\circledR}$ chromatographic manager system. Eluted constituents were identified by comparing the retention time with that of reference standards. For determination of phenolic compounds, the following reference standards were used: quercetin, kaempferol, astragalin, isoquercitrin, leucocyanidin, rutin, quercitrin, epicatechin, proanthocyanidin $\mathrm{A}_{2}$. The regression of the calibration curves of reference standards was linear, the coefficient of determination $\left(R^{2}\right)$ for all lines was $>0.9999$, and the resolution $\left(R_{\mathrm{s}}\right)$ of the standard peaks was $>1.5$. 
E. Zelbiene et al:: In vitro permeation studies of phenolics from horse chestnut seed gels prepared with different polyacrylic acid polymer derivatives, Acta Pharm. 65 (2015) 181-189.

\section{Evaluation of phenolic compounds}

The content of total polyphenols was determined by applying a modified Folin-Ciocalteau colorimetric method $(5,6)$. Following this method, $0.7 \mathrm{~mL}$ of the filtered test solution in dimethyl sulfoxide (DMSO) $\left(1 \mathrm{mg} \mathrm{mL}^{-1}\right.$ of extract) was transferred to a $10-\mathrm{mL}$ volumetric flask, the Folin-Ciocalteu reagent $(400 \mu \mathrm{L})$ was added, and after 3 minutes, sodium carbonate solution $\left(75 \mathrm{~g} \mathrm{~L}^{-1}\right)$ was added. After 2 hours, the suspension was centrifuged (at $5000 \mathrm{rpm}$ for 5 minutes), and the absorbance of the supernatant was measured at $760 \mathrm{~nm}$ (Thermo Scientific Genesys 20 Visible Spectrophotometer (Thermo Fiska Scientific, Inc., USA). The obtained linear equation $\left(R^{2}=0.9996\right)$ was used to evaluate the concentration of total polyphenols in the horse chestnut seed extract and to measure the amount of polyphenol compounds that permeated from the gels into the acceptor medium.

\section{RESULTS AND DISCUSSION}

To our knowledge, this is the first investigation that analyzes the diffusion of horse chestnut seed-derived phenolic compounds from gels prepared with different polyacrylic acid polymer derivatives. Also, no permeation studies have been conducted with the newgeneration Ultrez polymers. The studied dosage form (hydrogel) was selected because of its biocompatibility with the skin and skin-like mechanical properties ensuring a rapid transfer of the active ingredient through the stratum corneum to the target site (7).

Chemical composition of the pure species varieties of $A$. hippocastanum as obtained by Folin-Ciocalteau method and HPLC is shown in Table I and is similar to those mentioned in other reports on the qualitative composition of horse chestnut seeds (8). Evaluation of the total phenolic compound content revealed a high amount of phenolic compounds $(6.12 \%)$ in the horse chestnut seed extract.

In total, 20 gels of different composition were prepared for the study (Table II) to evaluate the influence of the polyacrylic acid polymer type, peppermint oil concentration and viscosity of the gels for phenolic compound permeation in vitro. Four different polyacrylic acid polymers were selected in gel formulations, namely, Carbopol Ultrez 10, Carbopol Ultrez 20, Carbopol 980, and Carbopol 940, which all differed in their physicochemical characteristics (9-11). Although the viscosity of gels CU-1 (Carbopol Ultrez 10) and CA-1 (Carbopol Ultrez 20) differed significantly from CR-1 (Carbopol 980) and CB-1 (Carbopol 940) gels (data not shown), viscosity was not related to phenolic compound release. Carbopol 980 and 940 demonstrated better permeation than Carbopol Ultrez 10 and 20 (Fig. 1). Furthermore, polyphenolic compounds were less readily released from the Carbopol Ultrez 10 gel matrix than from gels with other polymers (Fig. 1).

Peppermint oil fragrance, which according to literature (12), may be also used as permeation enhancer, was used as an additive in our gel formulations at different concentrations $(0.1,0.5,1.0$ and $1.5 \%$, Table II). The results showed that peppermint oil, at concentrations of $0.1,0.5$, and $1.0 \%$ slightly reduced viscosity in all the studied gel samples, whereas at a concentration of $1.5 \%$, it significantly reduced viscosity in polyacrylic acid polymer-based gels with Ultrez 10, Ultrez 20, and Carbopol 980 but not with Carbopol 940. Furthermore, our study showed that peppermint oil significantly reduced the permeation of phenolic compounds compared to the control group (without additive) $(p<0.05)$ and, in 
E. Zelbiene et al:: In vitro permeation studies of phenolics from horse chestnut seed gels prepared with different polyacrylic acid polymer derivatives, Acta Pharm. 65 (2015) 181-189.

Table II. Composition of gel samples with different polyacrylic acid polymer derivatives

\begin{tabular}{llcl}
\hline Sample & Polymer derivative (1 \%) & Peppermint oil (\%) & Typical gel composition \\
\hline CU-1 & Carbopol Ultrez 10 & 0 & \\
CU-2 & Carbopol Ultrez 10 & 0.1 & \\
CU-3 & Carbopol Ultrez 10 & 0.5 & \\
CU-4 & Carbopol Ultrez 10 & 1.0 & \\
CU-5 & Carbopol Ultrez 10 & 1.5 & \\
CA-1 & Carbopol Ultrez 20 & 0 & Polymer 1 \% \\
CA-2 & Carbopol Ultrez 20 & 0.1 & Dry extract of A. hippocastanum \\
CA-3 & Carbopol Ultrez 20 & 0.5 & Propylene glycol 15\% \\
CA-4 & Carbopol Ultrez 20 & 1.0 & Isopropyl alcohol 25 \% \\
CA-5 & Carbopol Ultrez 20 & 1.5 & Peppermint oil \\
CR-1 & Carbopol 980 & 0 & Water ad 100 \% \\
CR-2 & Carbopol 980 & 0.1 & \\
CR-3 & Carbopol 980 & 0.5 & \\
CR-4 & Carbopol 980 & 1.0 & \\
CR-5 & Carbopol 980 & 1.5 & \\
CB-1 & Carbopol 940 & 0 & \\
CB-2 & Carbopol 940 & 1.5 & \\
CB-3 & Carbopol 940 & 0.1 & \\
CB-4 & Carbopol 940 & 0.5 & \\
CB-5 & Carbopol 940 & 1.0 & \\
\hline
\end{tabular}

contrast to the literature data (13-15), did not act as a permeation enhancer (Fig. 1), most probably due to its reaction with active horse chestnut compounds or due to the low concentrations tested.

The amount of released phenolic compounds was influenced by the type of Carbopol (Fig. 1). The largest amounts of phenolic compounds released within 6 hours were observed in CA-1 gel samples. In CU-1, the diffusion of phenolic compounds after 6 hours was by $17.2 \%(p<0.01)$ lower than that observed in gels produced with Carbopol Ultrez 20. Even though gels prepared with Carbopol Ultrez 20 released the largest amounts of phenolic compounds, the inhibitory effect of peppermint oil on phenolic compound permeation in these gels was the strongest (Fig. 1b).

The flux of phenolic compounds from the horse chestnut seed extract containing gels through a semi-permeable membrane decreased with time in all 20 investigated formulations. The highest flux of polyphenolic compounds (50-60\%) was registered after 0.5 hours (data not shown). Table III shows that after 6 hours, the highest flux of polyphenolic com- 

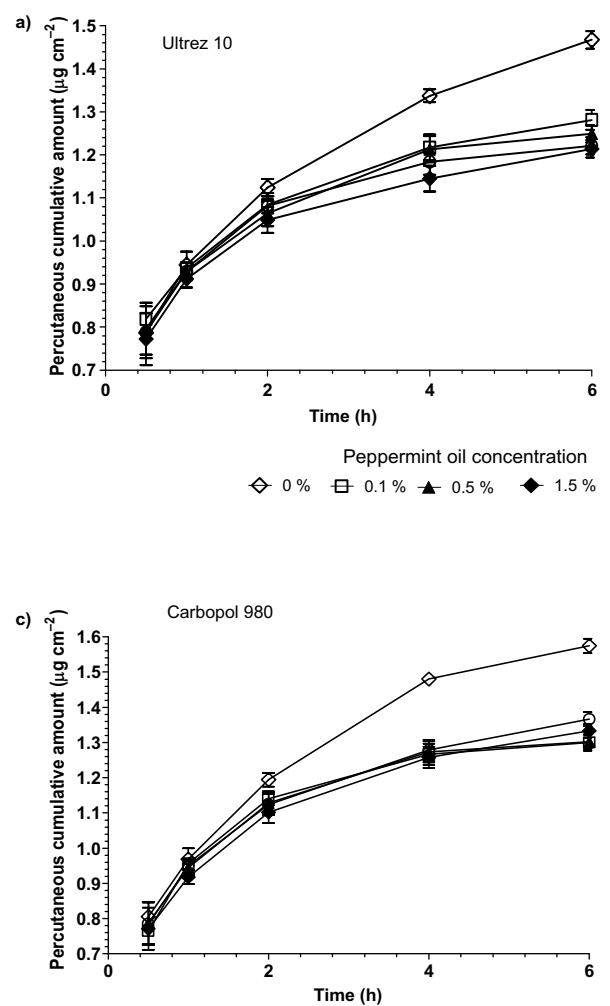

Peppermint oil concentration

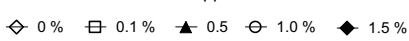

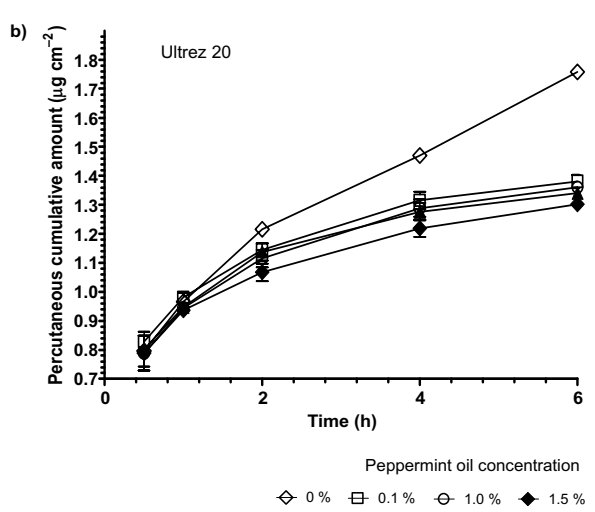

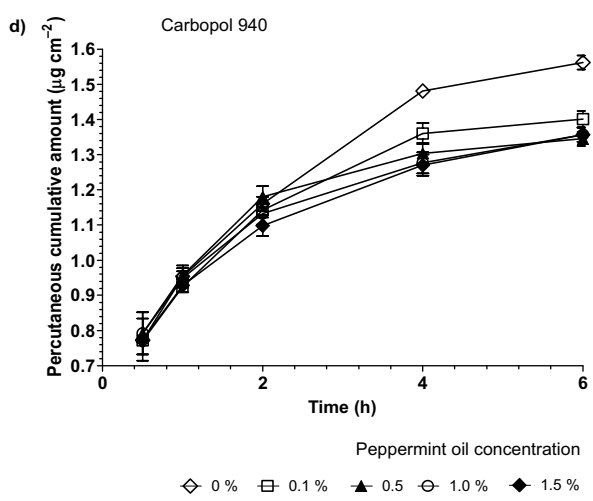

Fig. 1. The effect of peppermint oil $(0,0.1,0.5,1.0$ and $1.5 \%)$ on the permeation of horse chestnut seed extract-derived phenolic compounds from gels prepared using different polyacrylic acid polymer derivatives: a) Ultrez 10, b) Ultrez 20, c) Carbopol 980, d) Carbopol 940 (mean \pm SEM, $n=5$ ).

pounds was from gels prepared with Carbopol Ultrez 20, yet a significantly lower (by $9 \%$ ) flux of active compounds was observed only in gels prepared with Carbopol Ultrez 10 (compared to CA-1). The addition of peppermint oil into the gels significantly reduced the flux polyphenolic compounds from gels prepared with Ultrez 10, Ultrez 20, and Carbopol 980. In contrast to other gels, in formulations prepared with Carbopol 940, peppermint oil had no significant influence on the reduction of the flux of phenolic compounds at concentrations of $0.1,0.5$ or $1.0 \%$. Only the maximal peppermint oil concentration reduced the flux of the studied compounds by $7 \%(p<0.05 v s$. CB-1). The most effective permeation was observed in formulations produced with Ultrez 20 ( $p<0.01$, compared to other samples) (Table III).

In order to explain the mechanism by which different polymers affect phenolic compound permeation, percutaneous absorption parameters such as permeability coefficient, 
E. Zelbiene et al:: In vitro permeation studies of phenolics from horse chestnut seed gels prepared with different polyacrylic acid polymer derivatives, Acta Pharm. 65 (2015) 181-189.

Table III. Permeability parameters of different polymer based gels containing the horse chestnut seed extract

\begin{tabular}{|c|c|c|c|c|c|}
\hline Formulation $^{a}$ & $\begin{array}{c}\text { Flux } \\
\left(\mathrm{mg} \mathrm{cm}^{-2} \mathrm{~h}^{-1}\right)^{*}\end{array}$ & $\begin{array}{l}\text { Permeability } \\
\text { coefficient } \\
\left(\mathrm{cm} \mathrm{h}^{-1}\right)^{\mathrm{b}}\end{array}$ & $\begin{array}{l}\text { Enhancement } \\
\text { ratio }\end{array}$ & $\begin{array}{l}\text { Apparent } \\
\text { diffusion } \\
\text { coefficient } \\
\left(\mathrm{cm}^{2} \mathrm{~h}^{-1}\right)\end{array}$ & $\begin{array}{c}\text { Phenolic } \\
\text { compounds } \\
\text { after } 6 \mathrm{~h}(\% \text { of } \\
\text { applied dose })^{\mathrm{b}}\end{array}$ \\
\hline CU-1 & $2.287 \pm 0.002$ & $0.057 \pm 0.003$ & - & $4.335 \pm 0.010$ & $52.3 \pm 1.3$ \\
\hline CU-2 & $2.156 \pm 0.009$ & $0.065 \pm 0.002$ & $0.943 \pm 0.001$ & $3.307 \pm 0.009$ & $45.3 \pm 1.9$ \\
\hline CU-3 & $2.123 \pm 0.004$ & $0.067 \pm 0.004$ & $0.928 \pm 0.002$ & $3.143 \pm 0.009$ & $44.2 \pm 0.9$ \\
\hline CU-4 & $2.116 \pm 0.002$ & $0.068 \pm 0.001$ & $0.925 \pm 0.001$ & $3.004 \pm 0.011$ & $43.1 \pm 1.9$ \\
\hline CU-5 & $2.078 \pm 0.006$ & $0.069 \pm 0.003$ & $0.909 \pm 0.001$ & $2.970 \pm 0.007$ & $42.9 \pm 2.0$ \\
\hline CA-1 & $2.490 \pm 0.007$ & $0.047 \pm 0.008$ & - & $6.221 \pm 0.006$ & $63.1 \pm 2.3$ \\
\hline CA-2 & $2.282 \pm 0.009$ & $0.061 \pm 0.006$ & $0.916 \pm 0.002$ & $3.841 \pm 0.009$ & $48.9 \pm 2.1$ \\
\hline CA-3 & $2.223 \pm 0.004$ & $0.062 \pm 0.005$ & $0.893 \pm 0.003$ & $3.615 \pm 0.011$ & $47.5 \pm 1.7$ \\
\hline CA-4 & $2.217 \pm 0.007$ & $0.061 \pm 0.004$ & $0.890 \pm 0.001$ & $3.726 \pm 0.009$ & $48.3 \pm 1.8$ \\
\hline CA-5 & $2.157 \pm 0.005$ & $0.064 \pm 0.006$ & $0.866 \pm 0.004$ & $3.412 \pm 0.012$ & $46.1 \pm 2.5$ \\
\hline CR-1 & $2.392 \pm 0.009$ & $0.053 \pm 0.005$ & - & $4.990 \pm 0.009$ & $56.1 \pm 2.9$ \\
\hline CR-2 & $2.212 \pm 0.010$ & $0.064 \pm 0.003$ & $0.925 \pm 0.003$ & $3.404 \pm 0.012$ & $46.0 \pm 1.6$ \\
\hline CR-3 & $2.194 \pm 0.004$ & $0.064 \pm 0.002$ & $0.917 \pm 0.001$ & $3.411 \pm 0.007$ & $46.0 \pm 1.6$ \\
\hline CR-4 & $2.231 \pm 0.007$ & $0.061 \pm 0.005$ & $0.933 \pm 0.001$ & $3.760 \pm 0.004$ & $48.5 \pm 1.3$ \\
\hline CR-5 & $2.171 \pm 0.005$ & $0.063 \pm 0.006$ & $0.908 \pm 0.002$ & $3.576 \pm 0.005$ & $47.3 \pm 1.1$ \\
\hline CB-1 & $2.353 \pm 0.011$ & $0.053 \pm 0.004$ & - & $4.913 \pm 0.009$ & $55.8 \pm 1.3$ \\
\hline CB-2 & $2.233 \pm 0.004$ & $0.059 \pm 0.003$ & $0.949 \pm 0.002$ & $3.953 \pm 0.004$ & $49.8 \pm 1.2$ \\
\hline CB-3 & $2.256 \pm 0.003$ & $0.062 \pm 0.004$ & $0.959 \pm 0.004$ & $3.644 \pm 0.001$ & $47.6 \pm 1.5$ \\
\hline CB-4 & $2.230 \pm 0.005$ & $0.062 \pm 0.007$ & $0.948 \pm 0.001$ & $3.707 \pm 0.002$ & $48.1 \pm 1.3$ \\
\hline CB-5 & $2.191 \pm 0.007$ & $0.061 \pm 0.008$ & $0.931 \pm 0.002$ & $3.707 \pm 0.004$ & $48.2 \pm 1.1$ \\
\hline
\end{tabular}

Mean \pm SEM, $n=5$.

a For explanation of gel samples see Table II.

${ }^{\mathrm{b}} 0.5 \mathrm{~g}$ donor phase contains $154.5 \pm 9.0 \mu \mathrm{g}$ of polyphenolic compounds.

apparent diffusion coefficient, and enhancement ratio were calculated for the tested gel formulations (Table III). We found no significant differences in the permeability coefficient and apparent diffusion coefficient between the tested formulations (except for gels produced with Ultrez 20), but the partition coefficients differed. Thus, our results imply that the interaction between the active compounds in the horse chestnut seed extract, pepper- 
E. Zelbiene et al:: In vitro permeation studies of phenolics from horse chestnut seed gels prepared with different polyacrylic acid polymer derivatives, Acta Pharm. 65 (2015) 181-189.

mint oil and the gel-forming polyacrylic acid polymers could affect the ability of the phenolic compounds to partition from the vehicle into the stratum corneum, thereby influencing their permeation through the membrane.

\section{CONCLUSIONS}

The highest content of phenolic compounds $\left(1.758 \mu \mathrm{g} \mathrm{cm}^{-2}\right)$, compared to their content in the initial sample, penetrated in vitro from the polymer gel prepared using Carbopol Ultrez 20 ( $p<0.05$ vs. Carbopol Ultrez 10, Carbopol 980 ir Carbopol 940) without peppermint oil supplement. Our results showed that peppermint oil decreased both the viscosity of polymer gels and permeation of horse chestnut seed extract-derived phenolic compounds from all the polymer gels tested. Therefore, it could be suggested to avoid the use of peppermint oil as a fragrance or cooling agent when preparing polymer-based gels with the horse chestnut seed extract.

Further studies are needed for systematic investigations on three-dimensional human skin models.

\section{REFERENCES}

1. F. Bahri, R. Harrak, N. Achak and A. Romane, Chemical composition and antibacterial activities of the essential oils isolated from Juniperus thurifera L. var. Africana, Nat. Prod. Res. 27 (2013) 1789-1794; DOI: 10.1080/14786419.2012.755678.

2. I. Cvetkovikj, G. Stefkov, J. Acevska, J. P. Stanoeva, M. Karapandzova, M. Stefova, A. Dimitrovska and S. Kulevanova, Polyphenolic characterization and chromatographic methods for fast assessment of culinary Salvia species from South East Europe, J. Chromatogr. A 1282 (2013) 38-45; DOI: 10.1016/j.chroma.2012.12.068.

3. J. Arct and K. Pytkowska, Flavonoids as components of biologically active cosmeceuticals, Clin. Dermatol. 26 (2008) 347-357; DOI: 10.1016/j.clindermatol.2008.01.004.

4. J. A. Wilkinson and A. M. Brown, Horse chestnut - Aesculus hippocastanum: Potential applications in cosmetic skin-care products, Int. J. Cosmet. Sci. 21 (1999) 437-447; DOI: 10.1046/j.1467-2494.1999. 234192.x.

5. R. Masteikova, J. Bernatoniene, R. Bernatoniene and S. Velziene, Antiradical activities of the extract of Passiflora incarnata, Acta Pol. Pharm. 65 (2008) 577-583.

6. J. Bernatoniene, R. Masteikova, D. Majiene, A. Savickas, E. Kevelaitis, R. Bernatoniene, K. Dvorackova, G. Civinskiene, R. Lekas, K. Vitkevicius and R. Peciura, Free radical-scavenging activities of Crataegus monogyna extracts, Medicina (Kaunas) 44 (2008) 706-712.

7. S. A. Al-Suwayeh, E. I. Taha, F. M. Al-Qahtani, M. O. Ahmed and M. M. Badran, Evaluation of skin permeation and analgesic activity effects of carbopol lornoxicam topical gels containing penetration enhancer, Sci. World J. 2014 (2014) article ID 127495; DOI: 10.1155/2014/127495.

8. I. Kapusta, B. Janda, B. Szajwaj, A. Stochmal, S. Piacente, C. Pizza, F. Franceschi, C. Franz and W. Oleszek, Flavonoids in horse chestnut (Aesculus hippocastanum) seeds and powdered waste water byproducts, J. Agric. Food Chem. 55 (2007) 8485-8490.

9. N. A. Peppas, P. Bures, W. Leobandung and H. Ichikawa, Hydrogels in pharmaceutical formulations, Eur. J. Pharm. Biopharm. 50 (2000) 27-46.

10. N. A. Peppas and R. Langer, New challenges in biomaterials, Science 263 (1994) 1715-1720. 
E. Zelbiene et al.: In vitro permeation studies of phenolics from horse chestnut seed gels prepared with different polyacrylic acid polymer derivatives, Acta Pharm. 65 (2015) 181-189.

11. B. Narasimhan and N. A. Peppas, Molecular analysis of drug delivery systems controlled by dissolution of the polymer carrier, J. Pharm. Sci. 86 (1997) 297-304.

12. R. Rajan and D. T. Vasudevan, Effect of permeation enhancers on the penetration mechanism of transfersomal gel of ketoconazole, J. Adv. Pharm. Technol. Res. 3 (2012) 112-116; DOI: 10.4103/22314040.97286.

13. H. Tanojo, J. A. Bouwstra, H. E. Junginger and H. E. Bodde, In vitro human skin barrier modulation by fatty acids: skin permeation and thermal analysis studies, Pharm Res. 14 (1997) 42-49.

14. S. Gabbanini, E. Lucchi, M. Carli, E. Berlini and A. Minghetti, L. Valgimigli, In vitro evaluation of the permeation through reconstructed human epidermis of essentials oils from cosmetic formulations, J. Pharm. Biomed. Anal. 50 (2009) 370-376; DOI: 10.1016/j.jpba.2009.05.018.

15. S. Gu, J. Gao, X. Hou, B. Ding, W. Zhang, S. Gao and X. Ding, Effects of penetration enhancers on Shuangwu traumatic formula: In vitro percutaneous absorption and in vivo pharmacodynamic evaluation of an herb medicine, Eur. J. Pharm. Biopharm. 73 (2009) 385-390; DOI: 10.1016/j.ejpb.2009. 08.001. 